\title{
A Case of Chronic Gastric Anisakiasis Coexisting With Early Gastric Cancer
}

\section{Eiko Sakurai}

Fujita Health University

\section{Okubo Masaaki}

Fujita Health University

\section{Yutaka Tsutsumi}

Pathos Tsutsumi

\section{Tomoyuki Shibata}

Fujita Health University

\section{Tomomitsu Tahara}

Fujita Health University

\section{Yuka Kiriyama}

Fujita Health University

Ayano Michiba

Fujita Health University

Naoki Ohmiya

Fujita Health University

Tetsuya Tsukamoto ( $\square$ ttsukamt@fujita-hu.ac.jp )

Fujita Health University https://orcid.org/0000-0002-7502-8724

\section{Case Report}

Keywords: nematode, anisakiasis, stomach, gastric cancer

Posted Date: August 10th, 2021

DOI: https://doi.org/10.21203/rs.3.rs-762985/v1

License: (c) (i) This work is licensed under a Creative Commons Attribution 4.0 International License. Read Full License 


\section{Abstract}

Background: Anisakiasis is a parasitic disease caused by the consumption of raw or undercooked fish that is infected by anisakis third-stage larvae. In countries, such as Japan, Italy, and Spain, where people have a custom of eating raw or marinated fish, anisakiasis is a common infection. Although anisakiasis has been reported to occur in the gastrointestinal tract in several countries, reports of anisakiasis accompanied by cancer are rare.

Case presentation: We present the rare case of a 40-year-old male patient who had anisakiasis coexisting with mucosal gastric cancer. Submucosal gastric cancer was suspected on gastric endoscopy and endoscopic ultrasonography. After laparoscopic distal gastrectomy, granulomatous inflammation with anisakis larvae in the submucosa was pathologically revealed beneath mucosal tubular adenocarcinoma. Histological and immunohistochemical investigation showed cancer cells as intestinal absorptive-type cells that did not harbor mucin.

Conclusion: Anisakis larvae could have injected into the cancer cells selectively because of the lack of mucin in the cancerous epithelium. Anisakiasis coexisting with cancer is considered reasonable rather than coincidental.

\section{Background}

Health hazards resulting from the ingestion of raw food have distressed people across the world since ancient times. Anisakiasis is a type of food poisoning caused by the ingestion of raw fish infected with larval nematodes of the Anisakidae family. Approximately 20,000 cases of anisakiasis are reported in the world annually, and $>90 \%$ of them are from Japan. [1] Recently, the consumption of undercooked fish has become popular in other countries; thus, anisakiasis is becoming a more global disease. The recent development of transport systems that can deliver fresher fish to us have increased the prevalence of anisakiasis. In 1960, Van Thiel et al. [2] first described that this disease was caused by the anisakis larvae in the Netherlands.

There are three type of anisakis larvae, as follows: Anisakis simplex (Anisakis larvae type I), Anisakis physeteris (Anisakis larvae type II), and Pseudoterranova decipients. Among them, Anisakis simplex is the most common pathogenesis of anisakiasis.

The larvae of anisakis change their form four times ( $L 1-L 3$, and the adult worm) in their life. The adult worm lives in marine mammals (final host), such as wheals, seals, and dolphins. [3] Eggs are produced in the final host and excreted in seawater. These first-stage larvae (L1) become embryonated eggs and hatch into free-swimming larvae-the second-stage larvae (L2). Small crustaceans (first intermediate host) eat the L2 larvae, which develop into the third-stage larvae (L3). Infected crustaceans are ingested by fish and squid (second intermediate host). The L3 larvae are transferred from smaller fish to larger ones. 
Finally, marine mammals eat infected fish, and the L3 larvae mature into an adult worm. If humans consume infected fish in the raw or undercooked state, the human can be the incidental host. The L3 larvae enter the digestive duct and cause anisakiasis. The length of anisakis third-stage larvae is $20-30$ $\mathrm{mm}$.

The following four types of diseases are caused by anisakis larvae: gastric anisakiasis, intestinal anisakiasis, extraintestinal anisakiasis, and anisakis allergy. Gastric anisakiasis is the most common anisakiasis, which is caused by direct injection into the gastric mucosa and causes abdominal pain, nausea, and vomiting. The easy availability of endoscopy has enabled the identification of a large number of cases of gastric anisakiasis. Intestinal anisakiasis sometimes leads to extraintestinal anisakiasis if the larvae penetrate the intestinal wall. Anisakis allergy was first reported in 1990 in Japan. [4] The symptoms are described in various ways, such as urticaria, digestive symptoms (diarrhea, vomiting, or abdominal pain), and anaphylactic shock; allergen-specific immunoglobulin E detection is useful for establishing a diagnosis of anisakis allergy.

The symptoms of anisakiasis are classified into the following two types: acute and chronic anisakiasis. Patients with acute anisakiasis exhibit serious symptoms, such as severe abdominal pain, nausea, and vomiting. Chronic anisakiasis is asymptomatic or causes mild intermittent abdominal pain for several weeks. If patients have been previously sensitized by an anisakis antigen, acute and drastic symptoms occurred through immediate allergy. Chronic anisakiasis occurs in never-sensitized patients and exhibit foreign-body reaction in the pathological findings (Table 1). [5-7]

Here, we describe the case of a patient who had anisakiasis coexisting with early gastric cancer.

\section{Case Presentation}

A 40-year-old man with a gastric ulcer, which was diagnosed owing to a suspicion of gastric cancer in a mass screening, was referred to our hospital for further diagnostic evaluation.

Gastrointestinal endoscopy (Figure 1) revealed a submucosal tumor with a superficial ulcer on the posterior wall in the corpus. Irregular MV pattern (microvascular pattern) irregular MS pattern (microsurface pattern), and the demarcation line on the superficial depression were seen using M-NBI (magnifying endoscopy with narrow-band imaging). The lesion was diagnosed with early gastric cancer. The invasion to the submucosa was suspected on ultrasound endoscopy. Laparoscopic distal gastrectomy was performed. Full blood count and tumor markers were almost normal.

Shallow depression was found in the macroscopic view (Figure 2). Histologically, gastric cancer (welldifferentiated tubular adenocarcinoma) was found in the mucosa without submucosal invasion (Figures 2, 3A). The fact that the cancerous epithelium was positive for CDX-2 (Figure 3B) and CD10 (Figure 3C) proved that the epithelium was intestinal absorptive epithelium. The mucin core protein (MUC) of the cancer cells was negative for MUC5AC (Figure 3D), MUC6 (Figure 3E), and MUC2 (Figure 3F). PAS (Figure $3 \mathrm{G}$ ) and Alcian blue (Figure $3 \mathrm{H}$ ) were also negative, showing that the cancer cells lacked mucin. Ki-67 is 
aberrantly distributed (Figure 3I). Beneath the cancer, granulomas formed in the submucosal lesion (Figure 4A). Necrosis, severe neutrophilic and macrophages infiltration, and giant cells were observed in the granulomas (Figure 4B). There were few eosinophils in the area. Foreign material surrounded by the granuloma was suspected as the anisakis' digestive tract (Figure 4C). Immunohistochemical analysis was performed using monoclonal antibody, An2, against anisakis and visualized with 3,3'diaminobenzidine (Simple Stain-Max, Nichirei Bioscience Inc., Tokyo, Japan) and proved to be chronic gastric anisakis' granuloma (Figure 4D). [8]

The fact that the patient had no symptoms with development of foreign-body granuloma and a few eosinophils infiltrates implied this case was initial infection of anisakiasis. Considering these findings, the lesion was confirmed as intramucosal gastric adenocarcinoma that coexisted with submucosal granuloma caused by chronic anisakiasis.

\section{Discussion And Conclusion}

Most cases of gastric cancer accompanied by gastric anisakiasis have been reported in Japan. Sonoda et al. [9] discussed the cases of 30 patients who had anisakiasis associated with gastrointestinal cancer in Japan for 33 y (1970-2013). Twenty-three cases were those of patients with anisakiasis with gastric cancer, and the others were those of patients with anisakiasis with duodenal or colon cancer.

It is important to recognize that anisakiasis might lead to an incorrect clinical diagnosis. Murata et al. [10] reported the case of a patient with anisakiasis in the liver that mimicked metastatic liver cancer. It was pointed out that inflammatory granuloma formed in chronic anisakiasis might be confused with other granulomatous diseases, such as gastrointestinal tuberculosis and Crohn's disease. [5] Submucosal lesion formed by an anisakis larva could cause be misdiagnosed as submucosal tumor. [11] Submucosal granuloma also misled us about the preoperative depth of invasion. [12] In our case, the clinical diagnosis before the operation was that of submucosal cancerous lesion. After the operation, pathological findings revealed that it was mucosal gastric cancer with submucosal granulomatous inflammation accompanied by anisakiasis.

Some reports have shown that the coexistence of anisakis larvae and cancer is not coincidental. $[6,9,12-$ 15] Tsutsumi et al. [6] pointed out that the change in the mucin of cancerous epithelium made the anisakis larvae infest gastric mucosa easily. This case also suggests that anisakis larvae tend to inject into epithelium without gastric mucin. lino et al. [16] showed that anisakis larvae gathered around an artificial gastric ulcer and implied that the ulcer and cancerous lesion helped anisakis larvae to penetrate the mucosa more easily.

Hirotsu T, et al. [17]and Uozumi T et al. [18] described that wild-type C. elegans, a kind of nematode, show attraction to the urine or tissue of patients with cancer compared to those of controls.

Owing to the change in mucin, the scent of cancer, or some other reason, anisakis larvae might enter the cancerous lesion selectively, resulting in coexisting of the cancer and anisakiasis larvae infection. 


\section{Abbreviations}

HE: hematoxylin and eosin

CDX-2: caudal type homeobox2

CD10: cluster of differentiation 10

MUC: mucin core protein

PAS: Periodic acid-Schiff

\section{Declarations}

Ethical approval and consent to participate

The ethical approval and documentation for a case report was waived with approval of the Institutional Review Board at Fujita Health University.

Consent from the patient

Written informed consent was obtained from the patient for the publication of this case report and any accompanying images. A copy of the written consent is available for review by the Editor-in-Chief of this journal.

Availability of data and materials

There are no supporting data available.

Competing interests

The authors declare that they have no competing interests.

Funding

This study was supported by grant-in-aid for Scientific Research from Fujita Health University to TTs.

Authors' contributions

ES carried out histopathological evaluation and drafted the manuscript. TTs conceived of the study and participated in the design and the preparation of the manuscript. TTs, YK, and AM evaluated in histopathological findings. YT provided his expert histological and immunohistochemical insight regarding anisakis. MO, TS, TTa, and NO collected and evaluated and interpreted clinical data. All authors read and approved the final manuscript. 


\section{References}

1. Pravettoni V, Primavesi L and Piantanida M: Anisakis Simplex: Current Knowledge. Eur Ann Allergy Clin Immunol 2012, 44: 150-156.

2. van TP, Kuipers FC and Roskam RT: A Nematode Parasitic to Herring, Causing Acute Abdominal Syndromes in Man. Trop Geogr Med 1960, 12: 97-113.

3. Ishikura H Introduction. In: Ishikura H and Namiki M (Eds.), Gastric Anisakiasis in Japan, Epidemiology, Diagnosis, Treatment, Tokyo, Springer-Verlag, 1989: 1-2.

4. Kasuya S, Hamano H and Izumi S: Mackerel-Induced Urticaria and Anisakis. Lancet 1990, 335: 665.

5. Mineta S, Shimanuki K, Sugiura A, Tsuchiya Y, Kaneko M, Sugiyama Y, Akimaru K and Tajiri T: Chronic Anisakiasis of the Ascending Colon Associated with Carcinoma. J Nippon Med Sch 2006, 73: 169-174.

6. Tsutsumi Y and Fujimoto Y: Early Gastric Cancer Superimposed on Infestation of an Anisakis-Like Larva: A Case Report. Tokai J Exp Clin Med 1983, 8: 265-273.

7. Tsutsumi Y Anisakiasis. In: Tsutsumi Y (Ed.), Atlas of Infectious Disease Pathology, Tokyo, Bunkodo, 2000: 233-235.

8. Takahashi S, Ishikura H and Hayasaka H Monoclonal Antibody, Intradermal Reaction, and Sarles' Phenomenon. In: Ishikura H and Namiki M (Eds.), Gastric Anisakiasis in Japan, Epidemiology, Diagnosis, Treatment, Tokyo, Springer-Verlag, 1989: 103-111.

9. Sonoda H, Yamamoto K, Ozeki K, Inoye H, Toda S and Maehara Y: An Anisakis Larva Attached to Early Gastric Cancer: Report of a Case. Surg Today 2015, 45: 1321-1325.

10. Murata $Y$, Ando K, Usui M, Sugiyama H, Hayashi A, Tanemura A, Kato H, Kuriyama N, Kishiwada M, Mizuno S, Sakurai H and Isaji S: A Case of Hepatic Anisakiasis Caused by Pseudoterranova Decipiens Mimicking Metastatic Liver Cancer. BMC Infect Dis 2018, 18: 619.

11. Harima Y, Kono M, Tanimura T, Sugihara T, Murawaki Y, Miura M, Tanaka S, Yoshimura T and Yamada M: A Case of Gastric Anisakiasia Diagnosis as Gastric Submucosal Tumor (in Japanese). Jornal of Matue city hospital 2011, 15: 91-96.

12. Shinohara A, Yamamoto S, Sakamoto T, Ito J, Nishihara K, Miyata N and Oomori K: lia-Type Early Gastric Cancer Presenting as a Large Submucosal Tumor by the Underlying Submucosal Abscess (in Japanese). Stomach Intestine 1980, 15 (10): 1031-1035.

13. Saitoh K, Hashida H, Iwashiro N, Ohrara M, Ishizawa M, Kondo S and Kato H: A Case of lic Type Early Gastric Cancer Associated with Eosinophilic Granuloma Produces by Gastric Anisakiasis (in Japanese). J Jpn Surg Assoc 2004, 37(12): 1829-1833. 
14. Hata Y, Matskawa M, Nomura M, Yamashita K and Irie H: A Case of Early Gastric Cancer Associated with Eosinophilic Granuloma (in Japanese) Intern Med 1988, 62(4): 755-758.

15. Shinohara S, Ito Y, Hidaka Y, Sueo K and Katsura Y: A Case of Stomach Cancer and Coexistence of Gastric Anisakiasis (in Japasese). Jan J Clin Exp Med 1973, 48(5): 1218-1221.

16. Ino H, Tomioka T, Akaiwa J, Mochizuki S and So N: About Parasitic Granuloma, Anisakiasia and Anisakis-Like Larva of Stmach (in Japanese). Stomach and Intestine 1969, 4: 601-608.

17. Hirotsu T, Sonoda H, Uozumi T, Shinden Y, Mimori K, Maehara Y, Ueda N and Hamakawa M: A Highly Accurate Inclusive Cancer Screening Test Using Caenorhabditis Elegans Scent Detection. PLoS One 2015, 10: e0118699.

18. Uozumi T and Hirotsu T: [Development of an Early Cancer Detection Method Using the Olfaction of the Nematode Caenorhabditis Elegans] (in Japanese). Yakugaku Zasshi 2019, 139: 759-765.

\section{Tables}

Table 1. Acute and chronic anisakiasis

\begin{tabular}{lll} 
& Acute & Chronic \\
\hline Symptom & $\begin{array}{l}\text { Severe (abdominal pain, nausea, } \\
\text { vomiting) }\end{array}$ & $\begin{array}{l}\text { Asymptomatic or Mild (intermittent } \\
\text { abdominal pain) }\end{array}$ \\
\hline $\begin{array}{l}\text { Allergic } \\
\text { reaction }\end{array}$ & Immediate allergy & Not sensitized \\
\hline $\begin{array}{l}\text { Endoscopic } \\
\text { finding }\end{array}$ & $\begin{array}{l}\text { Anisakis larvae are found on mucosa, } \\
\text { edema, reddish, erosion }\end{array}$ & $\begin{array}{l}\text { Submucosal tumor (anisakis larvae are } \\
\text { found in submucosa) }\end{array}$ \\
\hline
\end{tabular}

Pathological Eosinophilic phlegmonous inflammation Foreign-body granuloma finding

\section{Figures}




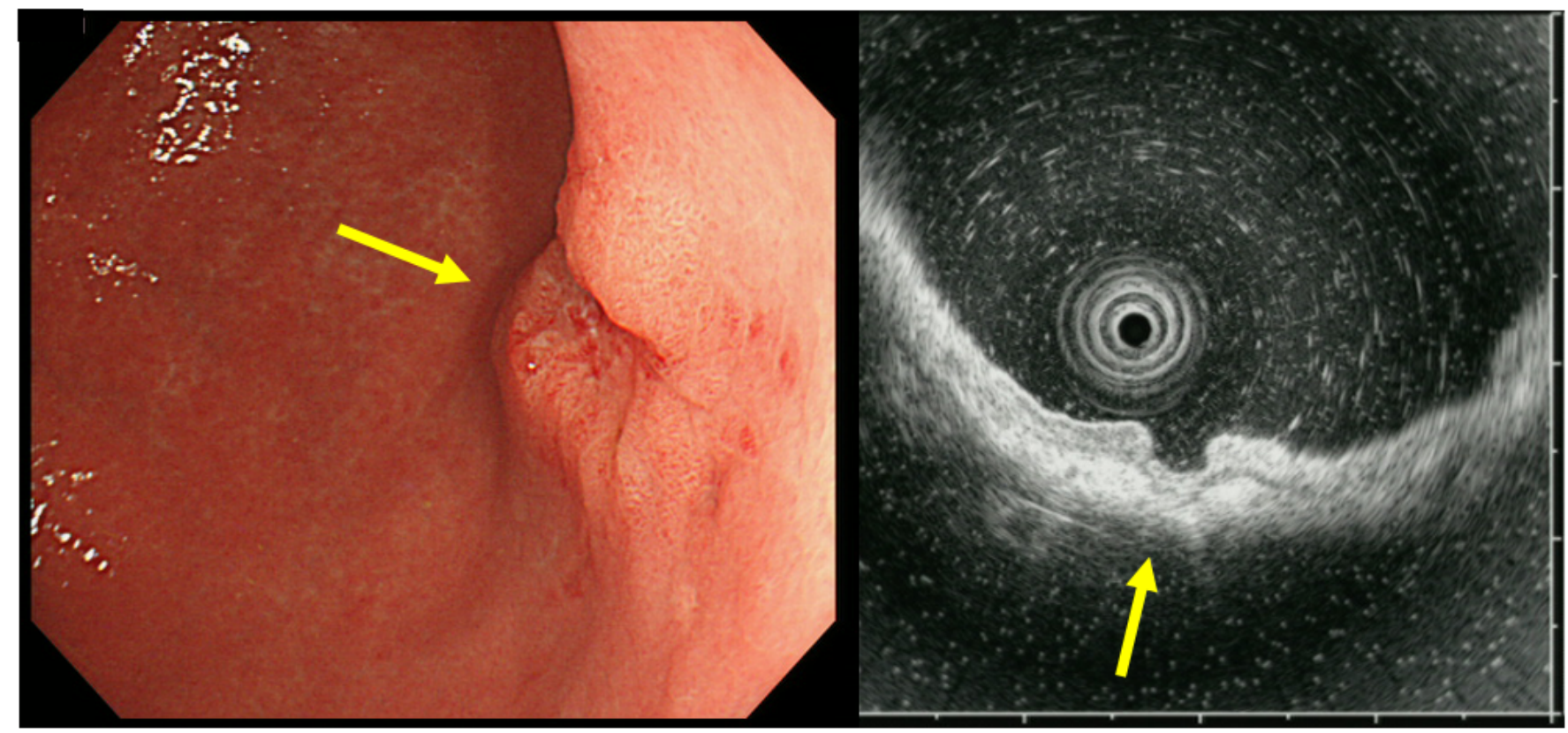

A

B

\section{Figure 1}

Gastric endoscopy and endoscopic ultrasonography. (A) Endoscopic analysis reveals superficial adenocarcinoma (arrow) on the posterior wall in the corpus. (B) In the endoscopic ultrasonography, the tumor was suspected to invade into the second layer (mucosa) with partial invasion into the third layer (submucosa) (arrow). 

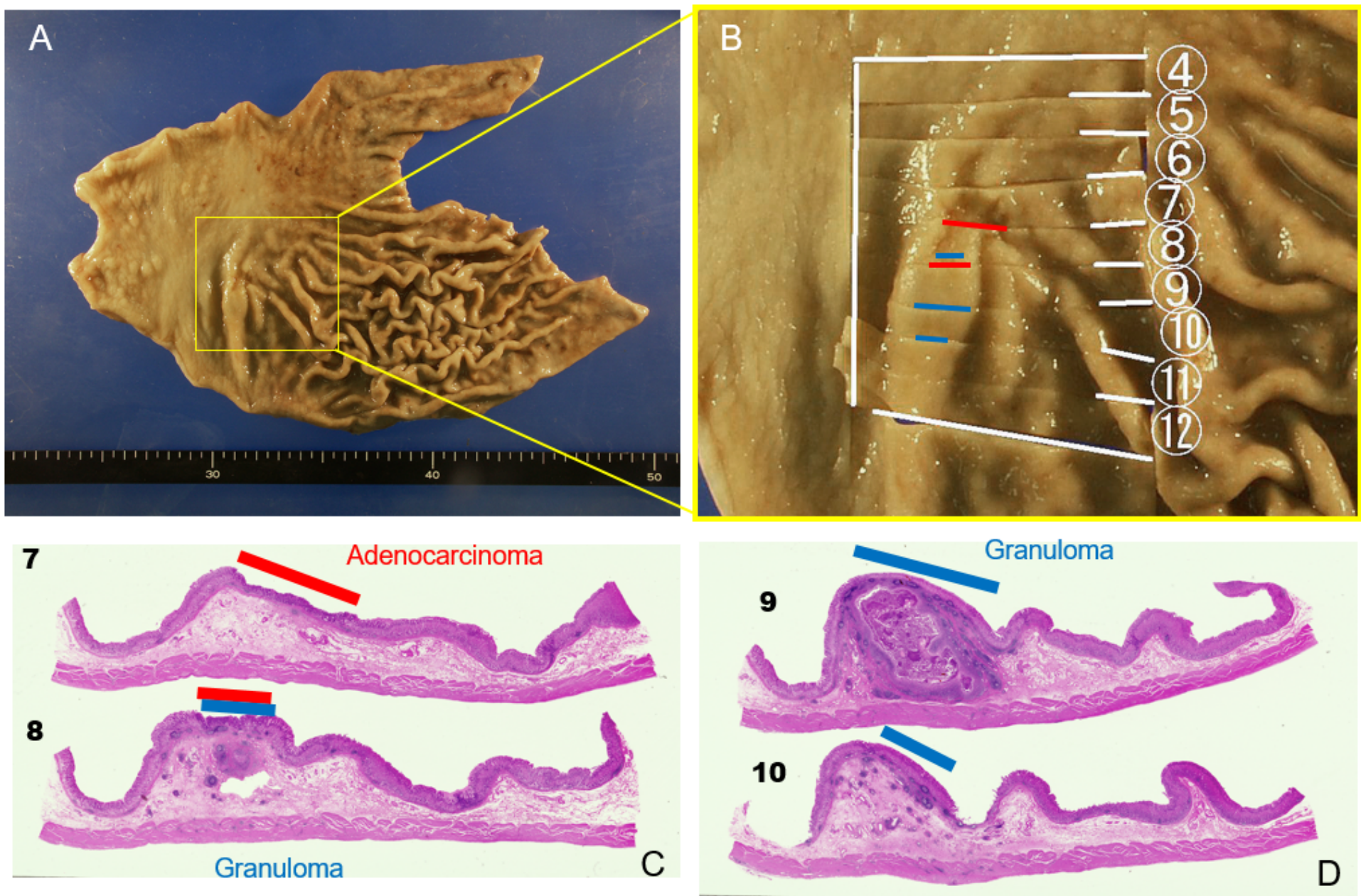

\section{Figure 2}

Macroscopic and loupe view. (A and B) Macroscopic view. Yellow square in A is enlarged to B. Shallow depression was found. ( $C$ and $D$ ) Submucosal granuloma (blue line) beneath the intramucosal adenocarcinoma (red line) is seen in the loupe view. 



\section{Figure 3}

Histological and immunohistochemical features of adenocarcinoma in the mucosa. (A) Hematoxylin and eosin staining. (B-F) Immunohistochemistry for cancer phenotype. Intestinal absorptive markers, CD10 (B) and CDX-2 (C). Mucin core proteins (MUCs), MUC5AC (D), MUC6 (E), and MUC2 (F). (G and H) Mucin histochemistry. Cancer cells are negative for PAS $(G)$ and Alcian blue $(H)$ mucin. Proliferative marker, Ki67 (I). Original magnification, $200 \times(A-I)$. 


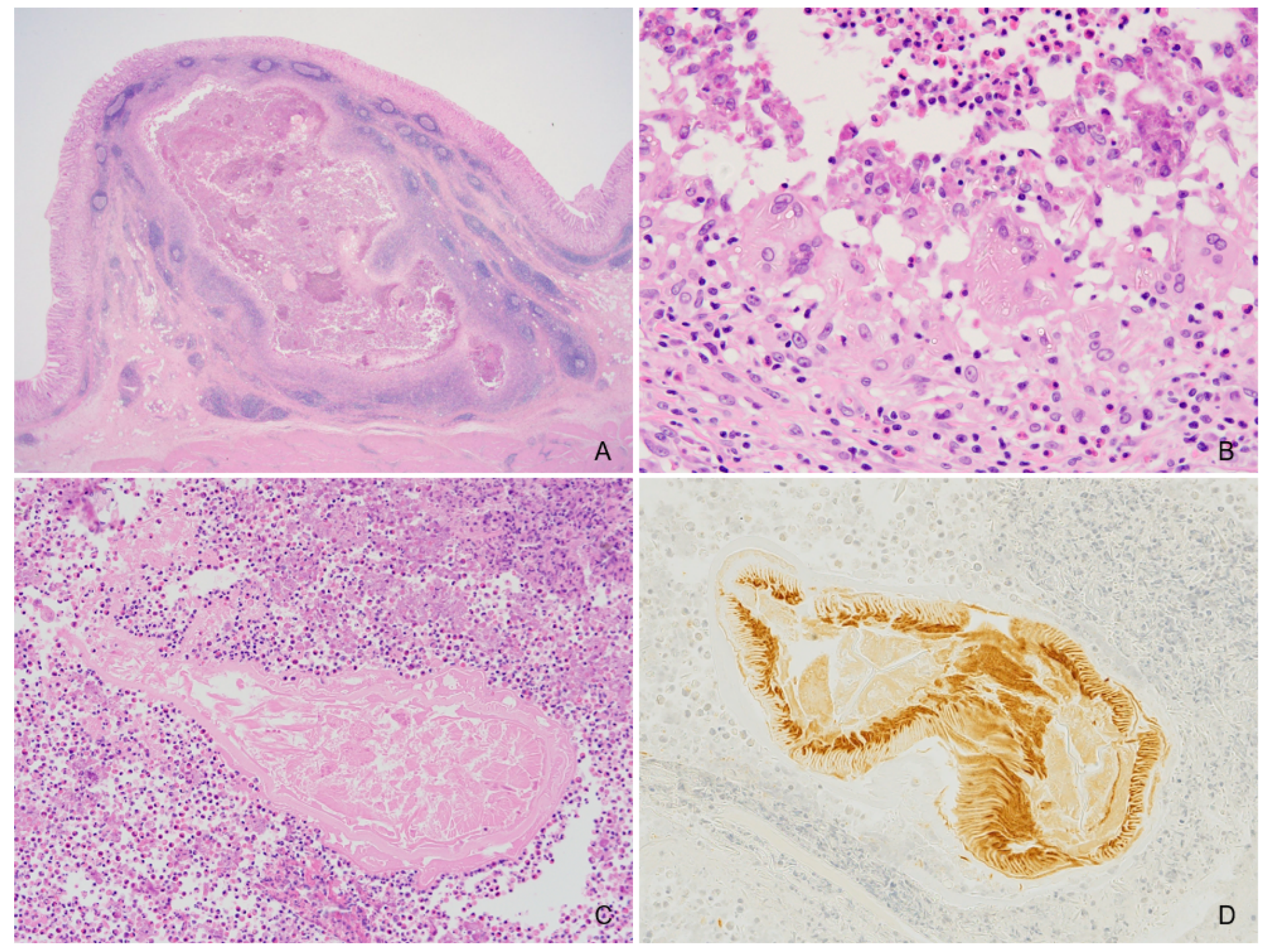

\section{Figure 4}

Granuloma. Submucosal lesion (A) was granuloma accompanied by necrosis, severe neutrophilic and macrophages infiltration, and emergence of the giant cells (B). Foreign material was surrounded by the granuloma (C) and was proved to be anisakis larvae immunohistochemically (D). Original magnification, $12.5 \times(A)$ and $200 \times(B-D)$.

\section{Supplementary Files}

This is a list of supplementary files associated with this preprint. Click to download.

- SakuraiCAREchecklist.pdf 